Disponible en ligne sur

ScienceDirect

www.sciencedirect.com
Elsevier Masson France

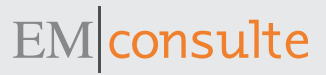

www.em-consulte.com

\title{
A systematic review on dry-land strength and conditioning training on swimming performance
}

\section{Effets de l'entraînement de force à sec sur le performance de nage : une revue systématique}

\author{
N.M. Amaro ${ }^{a, b}$, P.G. Morouço ${ }^{a, c, *}$, M.C. Marques ${ }^{b, d}$, \\ N. Batalha ${ }^{b, e}$, H. Neiva ${ }^{b, d}$, D.A. Marinhob,d
}

\footnotetext{
a Life Quality Research Centre, Polytechnic Institute of Leiria, Rua Dr. João Soares, 2400-448 Leiria, Portugal

${ }^{\mathrm{b}}$ Research Center in Sports Sciences, Health Sciences and Human Development, CIDESD, Quinta de Prados, 5001-801 Vila Real, Portugal

c Centre for Rapid and Sustainable Product Development, Polytechnic Institute of Leiria, Rua de Portugal, 2430-028 Marinha Grande, Portugal

d University of Beira Interior, Department of Sport Sciences, Convento de Santo António, 6200-001 Covilhã, Portugal

e University of Évora, R. Reguengos de Monsaraz, n' 14, 7000-727 Évora, Portugal
}

Received 16 June 2017; accepted 8 July 2018

\section{KEYWORDS}

Swimmers;

Performance;

Starts;

Turns;

Plyometric

\begin{abstract}
Summary
Objectives. - The objective of this review was to examine the effects of dry-land strength and conditioning $(\mathrm{S} \& \mathrm{C})$ training on swimming, and starts and turns performances.

News. - S\&C training is a common practice in swimming aiming to enhance performance or to prevent injuries. However, studies regarding the effects of S\&C on swimming performance are scarce; the influence of age, gender or competitive level is even scarcer.

Prospects and projects. - After a structured literature search, sixteen studies were included in the current review. Of those, seven did not report any positive or negative effects on swimming performance. Contrarily, most studies with positive effects were conducted with older swimmers whereas maximal strength was the most effective methodology for improving swimming performance. S\&C plyometric training is suggested to be the most effective method to
\end{abstract}

\footnotetext{
* Corresponding author. Centre for Rapid and Sustainable Product Development, Polytechnic Institute of Leiria, Rua de Portugal, 2430028 Marinha Grande, Portugal.

E-mail address: pedro.morouco@ipleiria.pt (P.G. Morouço).
} 


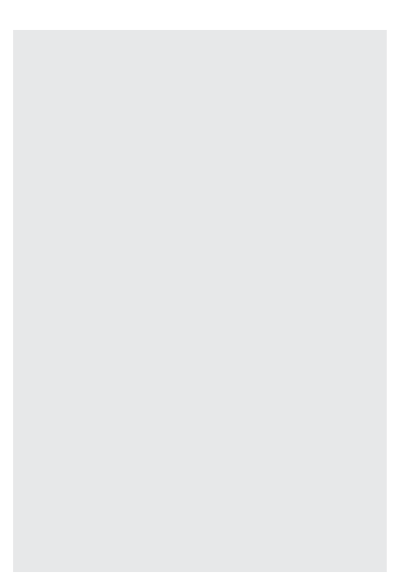

\section{MOTS CLÉS}

Nageurs ;

Performance ;

Départs ;

Virages ;

Plyométrique improve starts and turns. Future Randomized Controlled Trials should be conducted to explore the effects of S\&C induced by age and gender, on different swimming distances and techniques, and long-term training effects.

Conclusion. - It is recommended that S\&C training should be based on maximal strength, ranging from six to twelve weeks of 2 to 4 sessions per week (approximately 24 sessions altogether). In each session, coaches should vary from 2 to 3 sets and 3 to 5 repetitions, according to prescribed intensity. Rest intervals should range between 2 to 5 minutes and the intensity should be from 80 to $90 \%$ of 1 RM. Particularly regarding improving starts and turns, a S\&C training regime ranging from 6 to 8 weeks and with 2 sessions per week is suggested. In each session, swimmers should perform between 1 and 6 sets and 1 and 10 repetitions, according to the established intensity. Rest between sets should range from 60 to 90 seconds. The swimmers in the included studies are mostly men which do not allow to say if the recommendations made are gender-dependent. (c) 2018 Elsevier Masson SAS. All rights reserved.

\section{Résumé}

Objectifs. - L'objectif de cette révision a été d'examiner les effets de l'entraînement de force à sec sur les performances de nage, départs et virages.

Actualités. - L'entraînement de force à sec est une pratique commune en natation et a pour but d'augmenter la performance ou de prévenir les blessures. Pourtant, les études sur les effets de ce type d'entraînement sur la performance de nage restent encore peu nombreux ; l'influence de l'âge, le genre ou le niveau compétitif sont des questions encore moins abordées.

Perspectives et projets. - À la suite d'une recherche structurée, quinze études furent incluses dans cette révision. Parmi celles-ci, sept concluaient l'absence d'effet, positif ou négatif, sur la performance de nage. En revanche, en ce qui concerne les études qui mettent en avant les effets positifs sur la performance, l'entraînement de force maximum fût la méthodologie la plus performante, pour la plupart, chez les nageurs les plus expérimentés. L'entraînement plyométrique apparaît comme la méthodologie la plus performante pour améliorer la performance des départs et des virages. Les études futures devraient être menées pour explorer les effets de l'entraînement de force induits par l'âge et le sexe, sur les différentes distances et techniques de natation et les effets d'entraînement à long terme.

Conclusion. - Il est conseillé que l'entraînement de force à sec repose sur la force maximum, durant une période variant entre 6 et 12 semaines avec 2 ou 4 sessions hebdomadaires. Pour chaque session, les entraîneurs doivent réaliser entre 2 ou 3 séries et entre 3 ou 5 répétitions par série, tout en respectant l'intensité requise. Les intervalles de récupération doivent avoir une durée variable qui se situe entre 2 et 5 minutes, tandis que l'intensité devraient se situer entre les 80 et les $90 \%$ de $1 \mathrm{RM}$. Concernant l'amélioration des départs et des virages, il est suggéré que l'entraînement de force à sec varie entre les 6 et les 8 semaines avec 2 sessions hebdomadaires. Au cours de chaque session, les nageurs sont tenus de réaliser entre 1 et 6 séries et entre 1 et 10 répétions par série, tout en respectant l'intensité requise. Les intervalles de récupération doivent avoir une durée variable qui se situe entre 60 et 90 secondes. Les nageurs des études contemplées sont en majorité de hommes auxquels on défend de dire si les recommandations faites varient selon le sexe.

(c) 2018 Elsevier Masson SAS. Tous droits réservés.

\section{Introduction}

The ability to apply force in water is crucial in competitive swimming [1-5], particularly in short distances events $[6,7]$. As well as high values of strength and power, mostly in the upper body, have been identified as a determinant factor for success in competitive swimming [8-10]. Dry-land strength and conditioning (S\&C) training can improve swimming performance $[1,8,9,11-15]$, increase tethered swimming force $[8,16]$ and technical parameters such as increased stroke length $[11,14]$ and stroke rate [14]. Therefore, S\&C training is common in swimming prescription [17], being dry-land
$\mathrm{S} \& \mathrm{C}$ an alternative to in-water procedures, even if its specificity is questioned [18].

Coaches have prescribed S\&C training programs for decades to enhance swimming performance and/or prevent injuries [19-22]. Despite being a common practice, some coaches assume that S\&C training can negatively affect the swimmer's technical ability and consequently increase drag forces [5]. This is mostly due to the muscular hypertrophy and the decrease in flexibility commonly associated with S\&C training. Nevertheless, several improvements were associated with S\&C training, leading to an increase in maximum force, power 
and muscular endurance and optimization of performance $[5,23]$.

Studies examining the effects of S\&C on swimming performance have been conducted over the last 30 years, but its overall impact remains inconclusive $[16,18,24]$. While some of these investigations showed improvements in swimming starts and turns, and $25 \mathrm{~m}, 50 \mathrm{~m}, 400 \mathrm{~m}$ freestyle swimming races $[1,8,9,11-15]$, others did not $[10,16,18,24-27]$. The transferability of dry-land S\&C gains to swimming performance remains unclear and it is suggested to be a crucial factor for the absent of positive results.

The success of a S\&C program depends on several factors such as the type of training, methods, materials, periodization, and swimmers' maturation or competitive level. The optimal combination of these factors requires clarification and further investigation. In fact, few studies have focused on youth swimmers $[1,9,10,13,16,26]$, perhaps because of ethical issues [28]. Nevertheless, S\&C and in waterpower output seem to have a determinant influence on youth swimmers' performance and should be part of their training [29].

Hence, scientific research has not reached a consensus on the methodologies and benefits of S\&C training programs in swimming. The variability of research designs (e.g. protocols, outcomes selected, swimming events, and swimmers' competitive (evel) makes it difficult to compare data and come to practical conclusions. Therefore, the purpose of the present study was to examine possible effects of $S \& C$ training on swimming performance, as well as on starts and turns performances. A systematic review was done, summarizing evidence related to the effect of S\&C training on swimming performance. We are aware that the reduced number of studies conducted within this subject, associated to the lack of investigations with both sex, different ages, as well as more swimming techniques and distance could be an issue to clearly state a hypothesis. Nevertheless, we hypothesized that S\&C training will help swimmers to improve swimming velocity and starts and turns performance.

\section{Methods}

\subsection{Literature search}

An extensive literature search was conducted to identify studies from January 1st, 1985 until December 31st, 2017 in which S\&C training programs effects on swimming were investigated. This was done through computer searches (ISI Web of Knowledge, PubMed, Scopus and SPORTDiscus) using the keywords "swimming", "swimmer", "swim", "strength and conditioning", "strength", "strength training", "weight training", "resistance", "dry-land", "performance" and "longitudinal", with multiple combinations. In addition, the bibliographies of the located studies were extensively searched and cross-referenced. Those articles with restricted full text online were found in hardcopy form in library archives.

Studies selected for this review fulfilled the following selection criteria:

- the studies were written in English;

- they were published in a peer-reviewed journal;
- they contained research questions on the effects of S\&C training programs on competitive swimming;

- the main outcome reported was a performance measure (e.g. time or velocity);

- healthy human participants were used.

Review articles (qualitative review, systematic review, and meta-analysis) were not considered. The included studies focused on longitudinal interventions in S\&C training on competitive swimming. Studies based on other populations (e.g. paralympic swimmers) were excluded. Studies that did not present a complete description of their methods and/or results were excluded.

Our initial search identified 361 studies. After reading the titles and abstracts, sixteen articles were chosen for further analysis (Tables 1 and 2). Of these, four studies focused on the effects of S\&C training programs interventions on starts and turns performance and twelve studies on overall swimming performance. Those that were clearly not relevant or did not meet inclusion criteria were eliminated (Fig. 1).

\section{Results}

Tables 1 and 2 show the purpose, sample characteristics, S\&C training program, swimming performance measured and relevant findings. Several investigations showed improvements in $25 \mathrm{~m}$ [14], $50 \mathrm{~m}$ [1,11,14,15] and $400 \mathrm{~m}$ [8] freestyle swimming performance, in stroke length $[11,14]$, in stroke rate [14], in starts [9] and turns [13] performance.

Concerning samples, 8 in 16 studies did not randomly allocate subjects to group [8,10,12,14,18,24-26]. Other investigations did not include a control group [12,27]. Samples size ranged from 7 [1] to 38 subjects [26], with the age of participants being around 16 years old $(16.4 \pm 3.1$ years $)$. Seven studies assessed adolescent subjects $[2,9,13-16,27]$, six studies were conducted with young adults $[8,11,12,18,24,25]$ and only three studies assessed prepubescent swimmers $[1,10,26]$. In terms of gender, only one study focused exclusively on female swimmers [24], while nine studies evaluated male swimmers $[1,9,12,14,16,18,24,25,27]$. The remaining were conducted with mixed samples, coupling male and female subjects $[2,8,10,13,15]$ and only one compared the gender effect [8].

The intervention programs varied between four and twenty-four weeks, with six weeks being the most chosen length $(n=4)$. The frequency of sessions per week was between 2 and 4, and from 30 to 60 min per session. Volume per session varied between 10 and 36 repetitions. Sets varied from 2 to 3 sets per session. In studies using time instead of number of repetitions, the length of exercise execution varied from 10 to 120 seconds, with 2 to 6 sets per session. Intensity was expressed as a \% of $1 \mathrm{RM}$, ranging from 30 to $100 \%$ of $1 \mathrm{RM}+1 \mathrm{~kg}$. Some studies reported an intensity of exercises from 1 to $7 \mathrm{~kg}$ while others did not present intensities at all.

Weight lifting equipment was the most common material used $[2,8,10-12,15,18,25,27]$. Free weights were used in three studies $[14,18,24]$ and bodyweight exercises were used in seven studies $[1,2,9,10,12,13,15,26]$. Other materials such as medicine ball $[1,10]$, ergometer bicycle [27] and a hydro isokinetic ergometer [16] were also used. 
Table 1 Summary of the studies concerning the influence of S\&C programs on swimming performance.

\begin{tabular}{|c|c|c|c|c|}
\hline References & $\begin{array}{l}\text { Sample } \\
\text { Characteristics }\end{array}$ & $\begin{array}{l}\text { Dry-land S\&C } \\
\text { intervention }\end{array}$ & $\begin{array}{l}\text { Swimming } \\
\text { performance }\end{array}$ & $\begin{array}{l}\text { Findings in experimental } \\
\text { group }\end{array}$ \\
\hline Manning et al. [27] & $\begin{array}{l}\text { National level }(\delta) \\
\text { Experimental group } \\
(n=7,16.49 \pm 0.81 \\
\text { years) } \\
\text { No control group }\end{array}$ & $\begin{array}{l}\text { Power training (weight } \\
\text { lifting) } \\
9 \text { weeks ( } 3 \\
\text { sessions/week) } \\
2 \text { sets } \times \text { highest reps in } \\
1 \text { min } \\
\text { Weeks } 1-3: 30 \% \text {; weeks } \\
4-6: 40 \% \text {; weeks } 7-9: \\
50 \% 1 \text { RM } \\
11 \text { exercises }\end{array}$ & $\begin{array}{l}50,100 \text { and } 200 \text { yard } \\
\text { freestyle }\end{array}$ & $\begin{array}{l}50 \text { yard time: }-0.98 \mathrm{~s} \\
100 \text { yard time: }-0.06 \mathrm{~s} \\
200 \text { yard time: }-1.30 \mathrm{~s}\end{array}$ \\
\hline Strass [14] & $\begin{array}{l}\text { Competitive } \\
\text { swimmers } \\
(n=17 \delta ; n=2 \text { o }) \\
\text { Experimental group } \\
(n=10,16.6 \pm 1.2 \\
\text { years) } \\
\text { Control group }(n=9, \\
17.8 \pm 3.9 \text { years })\end{array}$ & $\begin{array}{l}\text { Heavy explosive } \\
\text { training with free } \\
\text { weights } \\
6 \text { weeks ( } 4 \\
\text { sessions/week) } \\
3 \text { sets } \times 3 \text { reps at } 90 \% \\
\text { of } 1 \text { RM } \\
2 \text { sets } \times 2 \text { reps at } 95 \% \\
\text { of } 1 \text { RM } \\
1 \text { rep at } 100 \% \text { of } 1 \text { RM } \\
1 \text { rep attempted at } \\
100 \% \text { of } 1 \mathrm{RM}+1 \mathrm{~kg} \\
\text { Exercise: elbow } \\
\text { extensions }\end{array}$ & $\begin{array}{l}25 \text { and } 50 \mathrm{~m} \text { freestyle } \\
\text { Stroke rate } \\
\text { Stroke length }\end{array}$ & 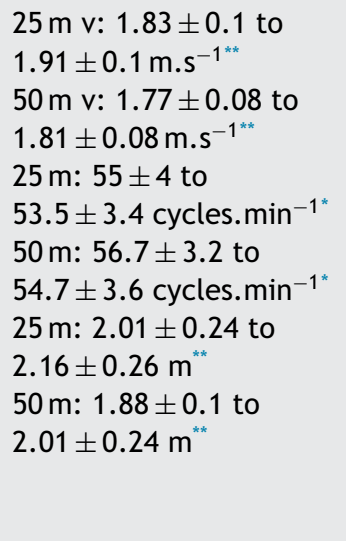 \\
\hline Tanaka et al. [18] & $\begin{array}{l}\text { Collegiate level }(\delta) \\
\text { Experimental group } \\
(n=12,19.17 \pm 0.32 \\
\text { years) } \\
\text { Control group }(n=12 \\
19.50 \pm 0.26 \text { years })\end{array}$ & $\begin{array}{l}\text { General strength } \\
\text { training (weight lifting } \\
\text { and free weights) } \\
8 \text { weeks ( } 3 \\
\text { sessions / week) } \\
3 \text { sets } \times 8 \text { to } 12 \text { reps } \\
\text { Exercises: dips, } \\
\text { chin-ups, lat pull } \\
\text { downs, elbow } \\
\text { extensions, bent arm } \\
\text { flys }\end{array}$ & $\begin{array}{l}22.9 \mathrm{~m} \text { freestyle } \\
365.8 \mathrm{~m} \text { freestyle } \\
\text { Stroke length }\end{array}$ & $\begin{array}{l}\text { No values reported } \\
\text { No differences between } \\
\text { groups for any measure }\end{array}$ \\
\hline Trappe \& Pearson [24] & $\begin{array}{l}\text { Collegiate level } \\
(3 ; n=10,20.1 \pm 1.2 \\
\text { years }) \\
\text { Weight assisted } \\
\text { training group - WAT } \\
(n=5) \\
\text { Free weight training } \\
\text { group - FWT }(n=5)\end{array}$ & $\begin{array}{l}\text { General strength } \\
\text { training (weight lifting } \\
\text { and free weights) } \\
6 \text { weeks ( } 2 \\
\text { sessions/week) } \\
\text { WAT: } 1 \text { set } \times \text { no weight, } \\
1 \text { set } \times 13.6 \mathrm{~kg}, 1 \\
\text { set } \times 22.7 \mathrm{~kg} \text { (until } \\
\text { failure) } \\
\text { Exercises: dips and } \\
\text { pull-ups } \\
\text { FWT: } 3 \\
\text { sets/exercise } \times 8-12 \\
\text { reps } \\
\text { Exercises: elbow } \\
\text { extension and flexion, } \\
\text { lat pull downs, bent } \\
\text { arm flys, quadriceps } \\
\text { extension, hamstring } \\
\text { flexion }\end{array}$ & $\begin{array}{l}22.9 \mathrm{~m} \text { freestyle } \\
365.8 \mathrm{~m} \text { freestyle } \\
\text { Stroke rate } \\
\text { Stroke length }\end{array}$ & $\begin{array}{l}\text { No differences between } \\
\text { groups for freestyle } \\
\text { velocity in } 22.9 \mathrm{~m} \text { and } \\
365.8 \mathrm{~m} \\
\text { No observed changes in } \\
\text { stroke rate and stroke } \\
\text { length }\end{array}$ \\
\hline
\end{tabular}


Table 1 (Continued)

\begin{tabular}{|c|c|c|c|c|}
\hline References & $\begin{array}{l}\text { Sample } \\
\text { Characteristics }\end{array}$ & $\begin{array}{l}\text { Dry-land S\&C } \\
\text { intervention }\end{array}$ & $\begin{array}{l}\text { Swimming } \\
\text { performance }\end{array}$ & $\begin{array}{l}\text { Findings in experimental } \\
\text { group }\end{array}$ \\
\hline Girold et al. [2] & $\begin{array}{l}\text { Regional/national } \\
\text { level } \\
(n=21 ; 10 \text { s, } 11 \text { \% }) \\
\text { Strength group: } \mathrm{S} \\
(n=7,16.5 \pm 2.5 \\
\text { years) } \\
\text { Assisted-sprint group } \\
(n=7) \\
\text { Control group ( } n=7 \\
16.5 \pm 1.5 \text { years })\end{array}$ & $\begin{array}{l}\text { Maximal strength } \\
\text { (weight lifting) } \\
12 \text { weeks ( } 2 \\
\text { sessions/week) } \\
\text { S group } \\
3 \text { sets } \times 6 \text { reps at } 80 \% \\
\text { to } 90 \% \text { of } 1 \text { RM } \\
\text { Exercises: press, } \\
\text { pull-up, draw with } \\
\text { barbells, abdominal, } \\
\text { squat and plyometric } \\
\text { jumps }\end{array}$ & $\begin{array}{l}50 \mathrm{~m} \text { freestyle } \\
\text { Stroke length } \\
\text { Stroke rate } \\
\text { Stroke depth }\end{array}$ & $\begin{array}{l}50 \mathrm{~m} \text { time: }-2.8 \pm 2.5 \%^{*} \\
1.61 \pm 0.11 \text { to } \\
1.59 \pm 0.09 \mathrm{~m} \\
48.9 \pm 4.98 \text { to } \\
50.7 \pm 3.71 \text { cycle. } \mathrm{min}^{-1} \\
0.86 \pm 0.05 \text { to } \\
0.83 \pm 0.05 \mathrm{~m}^{*}\end{array}$ \\
\hline Hong-Sun Song et al. [12] & $\begin{array}{l}\text { National level }(\delta) \\
\text { Experimental group } \\
(n=10 ; 18.50 \pm 2.07 \\
\text { years) } \\
\text { No control group }\end{array}$ & $\begin{array}{l}\text { Maximal strength and } \\
\text { power (weight lifting } \\
\text { and free weights) } \\
\text { 1st cycle }-18 \text { weeks (4 } \\
\text { sessions/week): } 10 \\
\text { weeks max. } \\
\text { strength + } 8 \\
\text { weeks-power } \\
\text { endurance } \\
\text { 2nd cycle }-20 \text { weeks } \\
\text { (4 sessions/week): } 12 \\
\text { weeks max. } \\
\text { strength + } 8 \\
\text { weeks-power } \\
\text { endurance }\end{array}$ & Swimming records & $\begin{array}{l}\text { 1st cycle: } 10 \text { personal } \\
\text { records, } 4 \text { Korean } \\
\text { records, } 2 \text { Asian records } \\
\text { 2nd cycle: } 8 \text { personal } \\
\text { records, } 4 \text { Korean } \\
\text { records, } 2 \text { Asian records }\end{array}$ \\
\hline Aspenes et al. [8] & $\begin{array}{l}\text { Competitive level } \\
\text { Experimental group } \\
(n=11,6 \delta, 5 \% ; \\
17.5 \pm 2.9 \text { years }) \\
\text { Control group }(n=9,2 \\
\delta, 7 \% ; 15.9 \pm 1.1 \\
\text { years) }\end{array}$ & $\begin{array}{l}\text { Maximal strength } \\
\text { (weight lifting) } \\
11 \text { weeks ( } 2 \\
\text { sessions/week) } \\
3 \text { sets } \times 5 \text { max. reps } \\
\text { Exercises: lat pull } \\
\text { downs }\end{array}$ & $\begin{array}{l}50 \mathrm{~m} \text { freestyle } \\
100 \mathrm{~m} \text { freestyle } \\
400 \mathrm{~m} \text { freestyle } \\
\text { Stroke length } \\
\text { Stroke rate }\end{array}$ & $\begin{array}{l}50 \mathrm{~m} \text { v: } 1.73 \pm 0.12 \text { to } \\
1.75 \pm 0.11 \mathrm{~m} . \mathrm{s}^{-1} \\
100 \mathrm{~m} \mathrm{v}: 1.59 \pm 0.1 \text { to } \\
1.61 \pm 0.1 \mathrm{~m} . \mathrm{s}^{-1} \\
400 \mathrm{~m} \mathrm{v}: 1.38 \pm 0.16 \text { to } \\
1.4 \pm 0.08 \mathrm{~m} . \mathrm{s}^{-1^{*}} \\
1.68 \pm 0.17 \text { to } \\
1.73 \pm 0.16 \mathrm{~m} \\
0.953 \pm 0.090 \text { to } \\
0.930 \pm 0.074 \mathrm{~Hz}\end{array}$ \\
\hline Garrido et al. [10] & $\begin{array}{l}\text { Competitive level } \\
\text { Experimental group } \\
(n=12,8 \approx, 4 \circ ; \\
12.0 \pm 0.78 \text { years }) \\
\text { Control group }(n=11, \\
6 \approx, 5 \circ ; \\
12.18 \pm 0.75)\end{array}$ & $\begin{array}{l}\text { Power and general } \\
\text { strength } \\
\text { Weight lifting } \\
8 \text { weeks ( } 2 \\
\text { sessions } / \text { week) } \\
2-3 \text { sets } \times 6-8 \text { reps at } \\
50 \text { to } 75 \% \text { of } 6 \mathrm{RM} \\
\text { Exercises: leg } \\
\text { extension; CMJ; CMJ to } \\
\text { box; medicine ball } \\
\text { throws ( } 1 \mathrm{~kg}) \text {; bench } \\
\text { press }\end{array}$ & $\begin{array}{l}25 \mathrm{~m} \text { freestyle } \\
50 \mathrm{~m} \text { freestyle }\end{array}$ & $\begin{array}{l}25 \mathrm{~m} \text { v: } 6.95 \% \mathrm{~m} . \mathrm{s}^{-1^{* *}} \\
50 \mathrm{~m} \text { v: } 4.77 \% \mathrm{~m} \cdot \mathrm{s}^{-1^{* *}} \\
\text { Control group } \\
25 \mathrm{~m} \text { v: } 6.44 \% \mathrm{~m} \cdot \mathrm{s}^{-1^{*}} \\
50 \mathrm{~m} \text { v: } 3.16 \% \mathrm{~m} \cdot \mathrm{s}^{-1^{*}}\end{array}$ \\
\hline Girold et al. [11] & $\begin{array}{l}\text { National level } \\
\text { Strength (S) group } \\
\left(n=8,4 \delta^{2}, 4 \text { + } ;\right. \\
21.1 \pm 1.4 \text { years }) \\
\text { Control group }(n=8,4 \\
\text { o, } 4 \text { \% } ; 24.2 \pm 4.6 \\
\text { years) }\end{array}$ & $\begin{array}{l}\text { Maximal strength } \\
\text { (weight lifting) } \\
4 \text { weeks ( } 3 \\
\text { sessions/week) } \\
3 \text { sets } \times 6 \text { reps at } 80 \text { to } \\
90 \% \text { of } 1 \text { RM } \\
\text { Exercises: pull-ups; lat } \\
\text { pull downs; draws with } \\
\text { pulleys }\end{array}$ & $\begin{array}{l}50 \mathrm{~m} \text { freestyle } \\
\text { Stroke length } \\
\text { Stroke rate }\end{array}$ & $\begin{array}{l}50 \mathrm{~m} \mathrm{v}: 2 \pm 1.3 \%^{*} \\
2.05 \pm 0.01 \text { to } \\
2.11 \pm 0.01 \mathrm{~m}^{*} \\
54.7 \pm 4.1 \text { to } \\
55.9 \pm 2.7 \text { cycle. } \mathrm{min}^{-1}\end{array}$ \\
\hline
\end{tabular}




\begin{tabular}{|c|c|c|c|c|}
\hline References & $\begin{array}{l}\text { Sample } \\
\text { Characteristics }\end{array}$ & $\begin{array}{l}\text { Dry-land S\&C } \\
\text { intervention }\end{array}$ & $\begin{array}{l}\text { Swimming } \\
\text { performance }\end{array}$ & $\begin{array}{l}\text { Findings in experimental } \\
\text { group }\end{array}$ \\
\hline Sadowski et al. [16] & $\begin{array}{l}\text { Competitive level ( }) \\
\text { Experimental group } \\
(n=14 ; 14.0 \pm 0.5 \\
\text { years) } \\
\text { Control group }(n=12 ; \\
14.1 \pm 0.5 \text { years })\end{array}$ & $\begin{array}{l}\text { Power (hydro isokinetic } \\
\text { ergometer) } \\
6 \text { weeks ( } 3 \\
\text { sessions / week) } \\
6 \text { sets } \times 50 \text { seconds of } \\
\text { work } \\
\text { Exercises: hydro } \\
\text { isokinetic ergometer }\end{array}$ & $\begin{array}{l}25 \mathrm{~m} \text { freestyle } \\
\text { Stroke length } \\
\text { Stroke rate }\end{array}$ & $\begin{array}{l}25 \mathrm{~m} \text { v: } 1.3 \% \\
\text { Performance: } 5.98 \% \\
\text { Performance: }-4.3 \%\end{array}$ \\
\hline Weston et al. [15] & $\begin{array}{l}\text { National level } \\
\text { Experimental group } \\
(n=10,5 \delta, 5 \div ; \\
15.7 \pm 1.2 \text { years }) \\
\text { Control group }(n=10 \\
5 \delta, 5+; 16.7 \pm 0.9 \\
\text { years) }\end{array}$ & $\begin{array}{l}\text { General strength } \\
12 \text { weeks ( } 3 \\
\text { session/week) } \\
2-3 \\
\text { sets } \times 30-120 \text { seconds } \\
\text { hold } \\
10-30 \\
\text { repetitions } \times 3-7 \mathrm{~kg} \\
\text { load } \\
\text { Exercises: prone } \\
\text { bridge; side bridge; } \\
\text { bird dog; leg raise; } \\
\text { overhead squat; sit } \\
\text { twist; shoulder press }\end{array}$ & $50 \mathrm{~m}$ freestyle & $\begin{array}{l}50 \mathrm{~m} \text { time: }-2.0 \% ; 4.2 \text { to } \\
1.1 \\
90 \% \text { confidence interval }\end{array}$ \\
\hline Amaro et al. [1] & $\begin{array}{l}\text { National level }(3) \\
\text { Sets/reps group }(\mathrm{Gr} 1) \\
(n=7,12.7 \pm 0.8 \\
\text { years) } \\
\text { Sets/time group (Gr2) } \\
(n=7,12.7 \pm 0.8 \\
\text { years) } \\
\text { Control group } \\
\text { ( } n=7.12 .6 \pm 0.8 \\
\text { years) }\end{array}$ & $\begin{array}{l}\text { Power } \\
6 \text { weeks }(2 \\
\text { session/week) } \\
\text { Gr1: } 3 \text { sets } \times 6-18 \text { reps } \\
\text { Gr2: } 3 \\
\text { sets } \times 10-25 \text { seconds } \\
\text { Exercises: medicine } \\
\text { ball throw down }(1 \mathrm{~kg}) \text {; } \\
\text { CMJ to box }(30 \mathrm{~cm}) ; \\
\text { dumbbell flys }(1.5 \mathrm{~kg}) \text {; } \\
\text { Russian twist }(3 \mathrm{~kg}) \text {; } \\
\text { push-ups }\end{array}$ & $50 \mathrm{~m}$ freestyle & $\begin{array}{l}50 \mathrm{~m} \text { time: } 33.43 \pm 2.83 \\
\text { to } 1.65 \pm 2.53 \mathrm{~s}^{* * * *} \\
\eta_{\rho}{ }^{2}=0.616\end{array}$ \\
\hline
\end{tabular}

These S\&C programs were implemented and their effects on swimming were tested by analyzing 25 yards [18,24], $25 \mathrm{~m}$ [10,13,14,16], 50 yard [27], $50 \mathrm{~m}[1,2,8,10,11,13-15], 100$ yard [27], $100 \mathrm{~m}$ [8], 200 yard [27], 400 yard [18,24] and $400 \mathrm{~m}[8,13]$ freestyle (front-crawl stroke) swimming performances. Only one investigation analyzed the effects on other swimming techniques [12] (Tables 1 and 2).

\section{Discussion}

\subsection{Effects of S\&C on swimming performance according to age}

Most investigations until now have been conducted with adolescents and young adults. In fact, only two investigations $[1,10]$ focused on prepubescent swimmers. The most recent study reported improvements of $2.21 \%$ in the $50 \mathrm{~m}$ front-crawl swimming time $\left({ }^{*}\right)$. Instead, no improvements were found in the previous study [10] being reported similar improvements in swimming velocity gains between experimental and control group (4.8 vs. $3.2 \%$, respectively). The lack of studies may be due to financial and ethical issues [29] or the assumption by coaches that technical training is more important than S\&C training $[8,10,28,30]$. Coaches usually assume that muscular hypertrophy and consequent decreases in flexibility may affect the swimmer's ability and increase drag forces [5]. Nevertheless, during the prepubescent stage, muscle hypertrophy is not believed to be the primary factor in strength improvement [31], with neuromuscular adaptations identified as the main explanation for strength gains [32,33]. It is indeed recommended that young athletes engage in resistance training, not only to enhance health, fitness and 
Table 2 Summary of the studies concerning the influence of S\&C programs on swimming performance (starts and turns).

\begin{tabular}{|c|c|c|c|c|}
\hline References & $\begin{array}{l}\text { Sample } \\
\text { Characteristics }\end{array}$ & $\begin{array}{l}\text { Dry-land S\&C } \\
\text { intervention }\end{array}$ & $\begin{array}{l}\text { Swimming } \\
\text { performance }\end{array}$ & $\begin{array}{l}\text { Findings in } \\
\text { experimental } \\
\text { group }\end{array}$ \\
\hline Cossor et al. [26] & 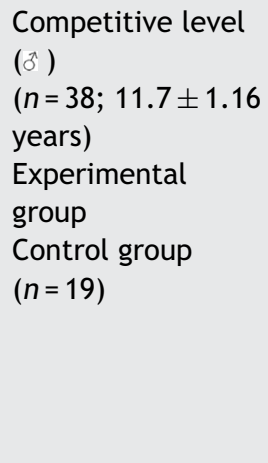 & $\begin{array}{l}\text { Plyometric } \\
20 \text { weeks ( } 3 \\
\text { sessions } / \text { week) } \\
2 \text { sets } \times 10-15 \\
\text { reps } \\
15 \text { exercises (not } \\
\text { described) }\end{array}$ & $\begin{array}{l}2.5 \mathrm{~m} \text { round trip } \\
5 \mathrm{~m} \text { round trip } \\
50 \mathrm{~m} \text { freestyle }\end{array}$ & $\begin{array}{l}2.5 \mathrm{~m} \text { time: } \\
3.58 \pm 0.39 \text { to } \\
3.32 \pm 0.42 \mathrm{~s} \\
5 \mathrm{~m} \text { time: } \\
7.45 \pm 0.72 \text { to } \\
6.94 \pm 0.67 \mathrm{~s} \\
50 \mathrm{~m} \text { time: } \\
38.46 \pm 3.36 \text { to } \\
37.51 \pm 2.9 \\
\text { No differences } \\
\text { between groups } \\
\text { for any measure }\end{array}$ \\
\hline Breed \& Young [25] & $\begin{array}{l}\text { Non-competitive } \\
\left(\begin{array}{l}(+) \\
n=23 ; 18.9 \text { years } \\
(s=1.5) \\
\text { Experimental } \\
\text { group }(n=12) \\
\text { Control group } \\
(n=11)\end{array}\right.\end{array}$ & $\begin{array}{l}\text { General strength } \\
\text { and power (weight } \\
\text { lifting) } \\
9 \text { weeks ( } 3 \\
\text { sessions/week) } \\
2-5 \text { sets } \times 4-10 \\
\text { reps of } \\
5 \text { RM-10 RM } \\
14 \text { exercises }\end{array}$ & $\begin{array}{l}\text { Flight distance } \\
\text { Take-off velocity } \\
\text { Take-off angle } \\
\text { Total horizontal } \\
\text { Imp } \\
\text { Horizontal Imp of } \\
\text { hands }\end{array}$ & $\begin{array}{l}\text { No improvements } \\
\text { TS: } 3.42 \pm 0.3 \text { to } \\
3.48 \pm 0.3 \mathrm{~m} . \mathrm{s}^{-1^{*}} \\
\text { TS: }-13.3 \pm 7.4 \text { to } \\
-10.1 \pm 5.6^{* *} \\
\text { TS: } 198 \pm 35.2 \text { to } \\
221 \pm 39.0 \mathrm{~N} . \mathrm{s}^{*} \\
\text { TS: } 65.6 \pm 32.7 \text { to } \\
94.0 \pm 41.2 \mathrm{~N} . \mathrm{s}^{* *}\end{array}$ \\
\hline Bishop et al. [9] & $\begin{array}{l}\text { Competitive level } \\
\text { Experimental } \\
\text { group }(n=11 ; \\
13.1 \pm 1.4 \text { years }) \\
\text { Control group } \\
(n=11 ; 12.6 \pm 1.9 \\
\text { years) }\end{array}$ & $\begin{array}{l}\text { Plyometric } \\
8 \text { weeks ( } 2 \\
\text { sessions/week) } \\
1-4 \text { sets } \times 1-5 \\
\text { reps } \\
\text { Intensity: low ( } 3 \\
\text { weeks); medium } \\
\text { ( } 3 \text { weeks); high ( } 2 \\
\text { weeks) } \\
19 \text { exercises }\end{array}$ & $\begin{array}{l}\text { Angle out of block } \\
\text { Distance to head } \\
\text { contact } \\
\text { Swim Block Start } \\
\text { velocity } \\
\text { Time to head } \\
\text { contact } \\
\text { Angle of entry } \\
\text { Time to } 5.5 \mathrm{~m}\end{array}$ & $\begin{array}{l}26.7 \pm 7.10 \text { to } \\
34.5 \pm 6.43^{* * * *} \\
1.70 \pm 0.19 \text { to } \\
1.83 \pm 0.19 \mathrm{~m}^{* * * *} \\
1.29 \pm 0.18 \text { to } \\
1.48 \pm 0.15 \mathrm{~m} . \mathrm{s}^{-1^{* * * *}} \\
1.32 \pm 0.09 \text { to } \\
1.24 \pm 0.06 \mathrm{~s}^{*} \\
42.3 \pm 7.33 \text { to } \\
47.5 \pm 3.95 \\
3.88 \pm 0.48 \text { to } \\
3.29 \pm 0.47 \mathrm{~s}^{* *+*}\end{array}$ \\
\hline Potdevin et al. [13] & $\begin{array}{l}\text { Competitive } \\
\text { swimmers } \\
\text { Experimental } \\
\text { group }(n=12,5 \AA, \\
7 \% ; 14.3 \pm 0.2 \\
\text { years) } \\
\text { Control group } \\
(n=11,5 \delta, 6 \circ ; \\
14.1 \pm 0.2 \text { years })\end{array}$ & $\begin{array}{l}\text { Plyometric } \\
6 \text { weeks ( } 2 \\
\text { sessions/week) } \\
1-6 \text { sets } \times 1-10 \\
\text { reps } \\
\text { Intensity: height } \\
\text { jump from } 0.21 \text { to } \\
1 \mathrm{~m} \\
15 \text { exercises }\end{array}$ & $\begin{array}{l}\text { Gliding velocity } \\
\text { Gliding } \\
\text { acceleration } \\
50 \text { m freestyle } \\
400 \text { m freestyle }\end{array}$ & $\begin{array}{l}2.28 \pm 0.19 \text { to } \\
2.41 \pm 0 \mathrm{~m} . \mathrm{s}^{-1^{*}} \\
\eta_{\rho}{ }^{2}=0.26 \\
6.80 \pm 1.12 \text { to } \\
4.81 \pm 0.90 \mathrm{~m} . \mathrm{s}^{-2^{* *}} \\
\eta_{\rho}{ }^{2}=1.99 \\
1.29 \pm 0.15 \text { to } \\
1.25 \pm 0.18 \mathrm{~m} \cdot \mathrm{s}^{-1^{*}} \\
\eta_{p}{ }^{2}=0.1 \\
0.96 \pm 0.09 \text { to } \\
0.92 \pm 0.10 \mathrm{~m} \cdot \mathrm{s}^{-1^{*}} \\
\eta_{\rho}{ }^{2}=0.15\end{array}$ \\
\hline
\end{tabular}

ऽ : male; : : female; CMJ: countermovement jump; Imp: impulse; TS: track start; $\eta_{\rho}{ }^{2}$ : effect size.

Indicates $P<0.05$.

** Indicates $P<0.01$.

*** $P<0.001$.

performance, but also to prevent sports-related injuries [32,33].

Studies conducted with adolescent swimmers showed that the effects of S\&C training may not be as clear as thought. Positive effects were reported in four studies
$[2,14,15,27]$ and no influence was reported in one study [16]. Within the adolescent age group, caution must prevail when analyzing results. Biological maturity is related to chronological age, and has a major impact on the physical performance of youth athletes [34]. Nevertheless, athletic 
361 papers located after excluding duplicates

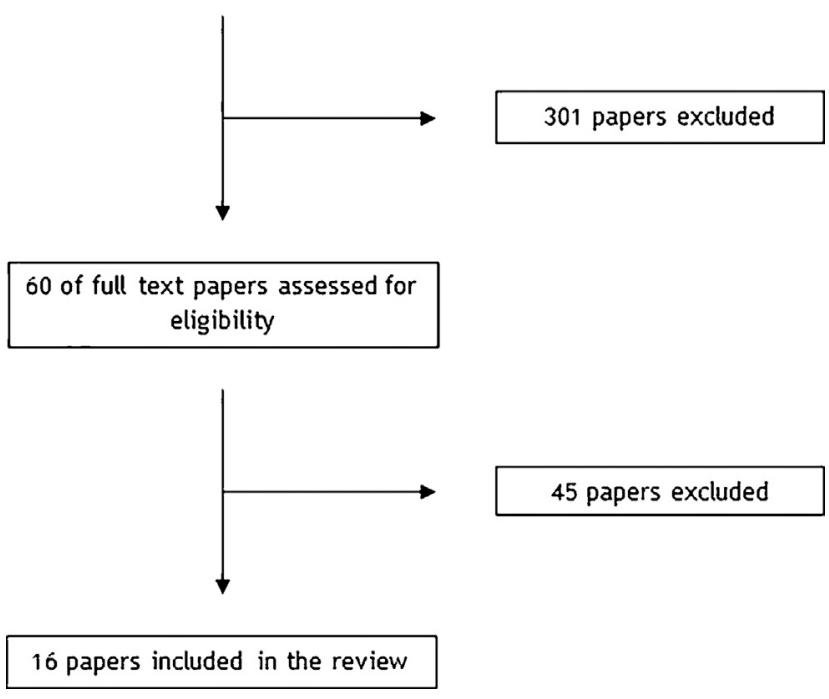

Figure 1 Flow of information through the different phases of paper selection for the systematic review.

performance may be influenced not only by training but also by growth and maturation [35], which can cause morphological and neural changes [36]. Maturity status was not provided in the aforementioned investigations. Therefore, with subjects ranging from 13 to 19 years old, and morphological characteristics being considered one of the main reasons for the differences in energetic profiles after puberty [37], the results may not be as clear as expected.

Three studies conducted with swimmers after puberty reported positive effects $[8,11,12]$ and no effects were reported in two other studies $[18,24]$. In both investigations where no positive effect was reported, the swimmers competed in collegiate teams. The lower competitive level of these swimmers could influence the results, since the energetic and biomechanical profiles of swimmers were different [28]. Swimming performance depends on the good relationship between bioenergetics and biomechanical parameters [38]. Thus, the transferability of dry-land strength gains to swimming performance could depend on the interaction of several parameters such as strength (dry-land and in-water) and biomechanics (kinematics and kinetic) [38], determined by competitive level.

Investigations have, so far, focused on adolescence and early adulthood. Investigations with age group swimmers must be conducted as a means of potentially enhancing swimming performance not only in the short term, but also in the long-term, and at different competitive levels.

\subsection{Effects of $S \& C$ on swimming performance according to gender}

As it occurs with competitive level, differences in muscular strength and anthropometrics emerge between genders, particularly after puberty $[37,39,40]$. However, there are few studies with samples separated according to gender. Results with mixed samples (male and female) must be analyzed with extra caution due to a possible gender effect. Even though during prepubescence strength improvements are quite similar between boys and girls [33], after this period boys have a tendency to exhibit higher muscle strength levels than girls $[41,42]$. Thus, coupling data from both sexes in research focused on S\&C may be misleading. Within the analyzed studies, only one study presented separated results between females and the whole group (male plus female) [8]. However, 7 out of 9 subjects were female in the control group and 5 out of 11 subjects were female in the intervention group, which may have led to heterogeneity between groups. For instance, the authors reported a significant correlation $(r=-0.975, P<0.01)$ between strength and $400 \mathrm{~m}$ swimming performance in the female group but not in the whole group results. The authors also reported significant improvement in tethered swimming force in both the female group and the whole group. On the other hand, in terms of swimming performance, only the whole group experienced significant improvements in the $400 \mathrm{~m}$ freestyle (-4 s mean). In adulthood differences in swimming performance between genders tend to be greater over short distances and less over higher distances [18]. Additionally, through having more fat mass than males, females can adopt a better body position, thereby increasing swimming economy [37]. By contrast, the remaining investigations with mixed samples did not present separated results, which may lead to misleading results. Yet, improvements in swimming time were reported in the $50 \mathrm{~m}[2,11,15]$. Whereas no significant differences between sexes were reported in two investigations [2,11], Weston et al. [15] attempted to balance groups by sex ( 5 males and 5 females). However, the results of the latter paper were coupled and so a gender effect could not be determined. The only study that did not report positive effects was conducted with age group swimmers [10], where differences in swimming performance between the sexes do not exist or tend to be non-relevant. Nevertheless, the study of heterogeneous groups in investigations of swimming performance can lead to ambiguous conclusions $[43,44]$.

Most of these studies were conducted with exclusively male samples $[12,14,16,18,24,28]$. Of those already mentioned, only two reported improvements in swimming performance $[12,14]$. On the other hand, there were no investigations conducted with an exclusively female sample up to the date. Thus, further investigations with separated samples must be conducted in order to draw conclusions on a possible gender effect. Yet, the available literature suggests a tendency for swimming performance to improve in investigations on the effects of S\&C training on mixed samples. Additionally, it is also crucial to cross compare information with age and consequently with the theoretical maturational development of swimmers.

\subsection{Effects of $S \& C$ on swimming performance according to training protocol}

Maximal strength training is the most regularly applied methodology in S\&C training programs. Furthermore, all investigations that have used maximal strength reported improvements of between 2 and $4 \%$ in swimming performance $[2,8,11,12,14]$. 
Six weeks of S\&C training with 4 sessions per week using intensities from 90 to $100 \%$ of 1 RM showed gains of between 20 and $40 \%$ of dry-land muscular strength [14]. These measurements occurred in isometric conditions that may not be related to swimming actions. Swimming actions are dynamic and isometric testing may therefore represent a lack of specificity [45]. Nonetheless, dry-land muscular strength gains allowed significant improvements in swimming performance over 25 and $50 \mathrm{~m}$ (4.4 and $2.1 \%$, respectively). In this investigation, only the upper body was exercised through elbow extension exercises and the use of free weights through maximal strength S\&C methodologies. Weight lifting equipment was used in the remaining investigations $[2,8,11,12]$.

S\&C training (S) based on maximal strength was applied for twelve weeks ( 2 sessions per week) and compared to resisted and assisted-sprint (RAS) training [11]. These authors found improvements in the $50 \mathrm{~m}$ time of the $\mathrm{S}$ $(2.8 \pm 2.5 \%)$ and RAS $(2.3 \pm 1.3 \%)$ groups compared to the control group $(0.9 \pm 1.2 \%)$. Improvements in muscle strength were more significant in the $\mathrm{S}$ group than in the RAS group. In contrast, the RAS group presented an increase in stroke rate in their $50 \mathrm{~m}$ freestyle performance. It was suggested that the application of S or RAS training was more effective than swimming training alone. These results were then corroborated by other researchers $[8,11]$. After eleven weeks ( 2 sessions per week) of combining maximal strength training and high intensity interval swimming training (HIIT) the results were positive in strength, tethered swimming force and $400 \mathrm{~m}$ freestyle performance [8]. However, no improvements occurred in short distance performance $(50 \mathrm{~m}$ or $100 \mathrm{~m}$ ) and biomechanical variables (stroke length and stroke rate). With the ability to exert force in the water a decisive factor over short distances $[6,7,17]$, it would be of great importance to clarify the lack of effect in sprint distances. Moreover, the positive effects on tethered swimming and $400 \mathrm{~m}$ freestyle were correlated in the female subjects of the intervention group. These results appeared to suggest that in-water force exertion may be important in middle swimming distances for female swimmers. However, it was not determined if the positive effects in swimming performance were a result of the combined methodologies or if a crossover between HIIT and S\&C sessions occurred. HIIT sessions were composed of $4 \times 4$ min of high intensity interval training. These efforts and durations may be better associated with the $400 \mathrm{~m}$ improvements.

An investigation compared the effects of a S\&C program (S) with those of an electrical stimulation (ES) program, both combined with a swimming program, over four weeks, on adult swimmers [11]. Additionally, the authors tried to verify if training effects lasted four weeks after the training period ended. The S\&C training sessions were performed 3 times per week. The authors reported an increase in swimming velocity for both $\mathrm{S}$ and $\mathrm{ES}$ group ( $2 \%$ and $1.7 \%$, respectively), at the end of the four-week intervention. Stroke length was increased only for the S group. Improvements in swimming velocity were maintained four weeks after the end of the program in both groups and no differences were reported between male and female swimmers. It seems that both methods were more efficient in improving swimming velocity than swimming training alone. However, electrical stimulation demands a higher investment from most swimming clubs than a S\&C program. Yet, this investigation only analyzed sprint performance ( $50 \mathrm{~m}$ freestyle) in high-level swimmers. Further investigations must be conducted with middle and long swimming distances as well as with swimmers operating at different competitive levels and with varying amounts of swimming expertise.

Interestingly, only four weeks and 12 sessions (18 repetitions per session) were sufficient to verify a positive effect in swimming performance. This volume was clearly less than that reported by other studies: 24 sessions with 15 repetitions per session [14], 24 sessions with 18 repetitions per session [2], 22 sessions and 15 repetitions per session [8], and 72 sessions in the first cycle of S\&C training and 80 sessions in the second cycle [12]. This may indicate that lower volumes and high intensity S\&C training may induce less neuromuscular fatigue and therefore improvements, leading to subsequent improved swimming performance.

S\&C training for a national team of male swimmers $(18.50 \pm 2.07$ years) was divided into two cycles applied according to the periodization of their main competitions (June and December) [12]. The first cycle lasted eighteen weeks with 4 sessions per week, emphasizing peak muscle strength, power and muscle endurance maintenance. The second cycle lasted for twenty weeks with 4 sessions per week of maximal strength, power and muscle endurance maintenance. Although several dry-land tests were performed, no swimming tests were performed to assess effectiveness of this S\&C training program. Yet, the authors reported 18 personal records, 8 Korean records and 3 Asian records in those two main competitions. Nevertheless, there was no statistical analysis of the influence of the S\&C training on swimming performance and there was no control group. Authors concluded that S\&C training program enhanced muscular functions and swimming performance, nevertheless with a significant decrease in flexibility. Although this was the investigation carried out over the largest number of weeks, the lack of swimming tests, some dubious procedures and reports (contradictory information on the number of subjects and weeks throughout the text) necessitates caution when analyzing its conclusions.

It is common to use the 1RM methodology to define the external load of exercises. However, when analyzing the influence of S\&C training in short distances, where power is crucial $[6,7,46,47]$, it is questionable whether $1 \mathrm{RM}$ is the force parameter with the higher association with power. For instance, the velocity with which exercises are performed at is crucial to increasing the specificity of S\&C exercises [48] and overall power output. S\&C training based on power (generation of force over a very short period of time) was applied in three investigations $[10,16,27]$. However, no positive effects in swimming performance were reported by any of those investigations. A S\&C training program based on speed and explosiveness (power) was applied to adolescent swimmers $(16.49 \pm 0.84$ years) [26]. Each training session consisted of 11 exercises (upper and lower body exercitation), performed in 2 sets of the maximum number of repetitions during one minute. This was performed 3 times a week over nine weeks, using weight lifting equipment and an ergometer bicycle. Intensities varied from 30 to $50 \%$ of $1 \mathrm{RM}$ with a progressive increase of $10 \%$ every three weeks. Although there were no significant differences, authors 
presented improvements of $-0.98 \mathrm{~s},-0.06 \mathrm{~s}$ and $-1.30 \mathrm{~s}$ for the 50, 100 and 200 yard tests, respectively. In swimming, these small improvements can be remarkable, particularly in short swimming distances.

A S\&C program of circuit training over six weeks (3 sessions per week), comprising 6 sets of 50 s exercitation and $10 \mathrm{~s}$ of rest on a specific hydro isokinetic ergometer device each session, led to improved tethered swimming propelling force but not different values of dry-land strength, stroke kinematics and swimming performance ( $25 \mathrm{~m}$ front-crawl) [16]. In spite of the effort to mimic underwater movement, it was concluded that S\&C training was not specific enough to improve swimming performance. Isokinetic conditions are not related to in-water actions that are performed with different velocities along the stroke. It is crucial that S\&C exercises and tests stimulate the muscles used in swimming and that muscle tension be related to in-water conditions [19]. In addition, only 3 swimmers were sprinters $(n=26)$, which could have influenced the results, as swimming performance was measured in a $25 \mathrm{~m}$ front-crawl test.

Another study on power training program was applied on prepubescent swimmers and combined dry-land S\&C training and aerobic swimming training [10]. The swimming training program was complemented with 2 sessions per week of S\&C training (bench press and leg extension, medicine ball throwing with $1 \mathrm{~kg}$, countermovement jump alone and with a $30 \mathrm{~cm}$ box) over eight weeks. Although dry-land improvements were reported, no swimming performance improvement was found. The authors suggested that swimmers' competitive level could affect performance improvements. Yet, a detraining period (S\&C training cessation and maintenance of swimming training) of six weeks showed that, although strength parameters remained stable, swimming performance still improved. It is reasonable to wonder if swimmers benefit from an adaption period to S\&C gains, while performing swimming training. The literature showed that there was no risk of losing strength and power during short cessation periods of a S\&C training program $[49,50]$, and the effect on maximal power was smaller than that observed for maximal force [51]. So, it seems reasonable to investigate whether specific in-water training can be useful in taking effective advantage of S\&C program improvements, after cessation [10]. This continuous stimulation could be the bridge between S\&C gains and swimming performance. In fact, a recent study with prepubescent swimmers [1] found improvements in $50 \mathrm{~m}$ front-crawl swimming time only 4-week after the end of S\&C training program. Authors added a 6-week S\&C training program to the normal swimming training program. The program consisted on 2 sessions per week of S\&C (medicine ball throw down $-1 \mathrm{~kg}$; CMJ to box $-30 \mathrm{~cm}$; dumbbell Flys $-1.5 \mathrm{~kg}$; Russian twist $-3 \mathrm{~kg}$; push-ups) with two different methodologies. One group performed exercises following a set/repetition methodology with no restrictions on the time of execution. The other experimental group followed an explosiveness methodology, where subjects had to perform as many repetitions as they could in a specific time. Although dry-land improvements were found for both groups, no swimming improvements were reported at the end of S\&C training program (6-week). However, authors allowed a 4week adaptation period, where swimmers had their normal swimming training only. After this period, only the group that performed the $\mathrm{S} \& \mathrm{C}$ training program based on explosiveness presented a $2.21 \%$ improvement in $50 \mathrm{~m}$ swimming time. This fact may reinforce the importance of allowing swimmers to adapt to new dry-land strength improvements. Nevertheless, further investigations with different ages, competitive levels and post-evaluation periodization must be conducted to clarify this issue.

Finally, three studies based their investigations on different protocols from those already discussed. S\&C training with weight lifting equipment and free weights was conducted over eight weeks (3 sessions per week) [18]. Even though volume per session was presented ( 3 sets of $8-12$ repetitions), intensity was not. The authors only reported an increment of $25-35 \%$ in the resistance used over the eight weeks of S\&C. There were no improvements in swimming performance after the S\&C training period, possibly due to the overload of a cycle of competitions during the S\&C training. The potential lack of control of the load and swimming competitions could have jeopardized results. However, the authors concluded that the lack of specificity of S\&C training in relation to in-water actions (swimming) was the main reason for the absence of positive transferability of strength gains. These results were later corroborated [24] through the comparison of S\&C weight assisted training (WAT) and S\&C free weights training (FWT). The investigation was carried out over six weeks (2 sessions per week). In every session both groups were instructed to reach volitional fatigue (WAT) and exhaustion (FWT). No differences in short $(22.9 \mathrm{~m})$ and medium $(365.8 \mathrm{~m})$ distance swimming performance were found between experimental groups. However, results from this study may be misleading, since there was no control group and groups were composed of only a small number of swimmers (5 in each group). In addition, S\&C training was implemented over six weeks out of a total of twelve weeks follow-up, and the evaluation was performed in weeks 4 and 12. These confounding factors did not allow for a consistent interpretation of results.

Recently, an investigation tried to quantify the effects of an isolated core-training program on $50 \mathrm{~m}$ front-crawl [15]. A S\&C program was implemented 3 times per week over twelve weeks and included exercises which aimed to work out the lumbopelvic complex and upper region extending to the scapula. Each session comprised isometric (prone bridge and side bridge) and dynamic (bird dog; leg raise; overhead squat; sit twist and shoulder press) exercises. Every two weeks, volume per session was increased and varied between 60 s to 360 s hold; 30 to 90 repetitions and 3 to $7 \mathrm{~kg}$ of load. A large beneficial effect on $50 \mathrm{~m}$ swim time $(-2.0 \%$; $90 \%$ Confidence interval -3.8 to $-0.2 \%$ ) was found after the training period. Good core stability is supposed to have a positive influence on the efficient relationship of force production between upper and lower limbs [52]. A question to be raised relies on the isometric conditions of most included exercises in S\&C training. Swimming actions are dynamic and so it was expected that swimming specificity could be negatively affected. To the best of our knowledge, this is the first investigation on the effects of S\&C core training on swimming performance and further investigation is needed.

In a brief analysis, it seems that maximal strength is the most effective methodology for improving performance, mainly in short distances. However, different low-volume training programs seem to induce positive effects. In 
adolescent swimmers, S\&C training ranging from six to twelve weeks and with 2 to 4 (approximately 24 sessions) sessions per week, is suggested to improve swimming performance. In adulthood and at a high competitive level, a S\&C training program of four weeks ( 3 sessions per week) is suggested to improve swimming performance. In each session, the volume should vary from 2 to 3 sets of 3 to 5 repetitions, according to the chosen level of intensity. Rest intervals should vary between 2 to 5 minutes. Concerning the intensities, high velocities and loads ranging from 80 to $90 \%$ of $1 \mathrm{RM}$ are associated with improvements. Nevertheless, there should be caution when applying S\&C programs near competitions or over high volumes of swimming training, in order to avoid overreaching and overuse injuries. S\&C based on maximal strength requires adjustments in swimming to avoid overloading of the peripheral and central fatigue mechanism [53]. Most of the studies reporting improvements used weight lifting equipment that may not be affordable for many swimming clubs due to financial constraints. With regard to prepubescent swimmers, there were contradictory results regarding effects in swimming performance associated with S\&C training programs. Nevertheless, S\&C training based on explosiveness reported improvements in swimming performance in this age group. However, conclusions are not easy to draw as many confounding factors seem to exist in the available investigations.

\subsection{Effects of S\&C on starts and turns performance}

Starts and turns are explosive actions usually associated by coaches and swimmers with the strength of the swimmer or to dry-land S\&C training, despite the scarcity of scientific evidence. These actions in swimming require high values of power output $[54,55]$. Therefore, explosiveness is usually thought of as the main aim of S\&C training and supports the greater use of plyometric S\&C training. In fact, three of the four investigations analyzed the use of plyometric training to improve starts and turns performance $[9,13,26]$. Positive effects of plyometric training were reported in two studies $[9,13]$, while the third did not find a positive result [26].

A study examined the effects of a plyometric training program on freestyle tumble turns, in age group male swimmers [26]. The subjects performed from 300 to 450 ground contacts per session, 3 times per week, over twenty weeks. There were no significant differences between experimental and control groups, for any measure. Despite the adoption of low to moderate intensities, one can assume that overload may have impaired results. Moreover, the control group performed 90 minutes of swimming training and the experimental group performed 75 minutes, adding 15 minutes of $\mathrm{S \& C}$ training. Although there were no significant differences between the experimental and control group, in most of the parameters assessed, improvements (\%) in the control group were higher than those in the plyometric group. Moreover, no differences in turning performance were found between swimmers who attended fewer S\&C sessions (<49\%) when compared to those who attended more $S \& C$ sessions (> 75\%). This raises questions over the efficiency of a plyometric program, in early ages, and indicates that swimming training seems to be enough to improve freestyle turns. Moreover, the authors claimed that some maturational and growth changes could positively influence performance $[35,36]$, rather than plyometric training.

Plyometric training program was also tested with regard to starts, showing some positive results $[9,13]$. First, an investigation aimed to identify the effect of plyometric training, when added to habitual training regimes, on swim start performance [9]. Significant improvements were found between baseline and post-evaluations for plyometric training group when compared to the control group, in the time taken to reach $5.5 \mathrm{~m}(-0.59 \mathrm{~s}$ vs. $-0.21 \mathrm{~s} ; P<0.01)$ and velocity of take-off to water contact $\left(0.19 \mathrm{~ms}^{-1}\right.$ vs. -0.07 $\left.\mathrm{ms}^{-1} ; P<0.01\right)$. These results can be determinant for a race performance; however, there should be some caution in assessing these effects as no information was provided regarding maturation level or even the sample's gender. The latter investigation mentioned [27] corroborated these positive results [9], presenting effects of the maturational status (Tanner stages of 3 and 4 ) and finding no differences between groups. Investigations were carried out over eight [9] and eleven [27] weeks, with total volume per session higher in the most recent one [27]. Both investigations increased the intensity from low to high, increased the height, from 0.22 to $0.79 \mathrm{~m}$ [9], and from 0.21 to $1 \mathrm{~m}$ [27], and increased the number of jumps per session from 37 to 192 [9], and from 100 to 264 [27].

Improvements reported in the 50 and $400 \mathrm{~m}$ front-crawl velocity [27], suggest a positive influence on starts and turns within overall swimming performance. This positive influence was, according to the authors, explained by the significant correlation between improvements in Squat Jump (SJ) and velocity in $50 \mathrm{~m}$ front-crawl. As SJ mechanical and muscular requirements are similar to those of starts, the authors concluded that improvements in swimming velocity were due to this phase of the race. Despite this idea of "transferability", there were no specific results on starts and turns of the swimmers.

Only one study on starts was focused on power and general strength training [25]. The goal was to improve vertical jump ability, on the grab, swing and rear-weighted track starts in swimming. For that purpose, 16 exercises were performed over nine weeks. Vertical jump was emphasized, as well as upper and lower body strength and power maintenance. The training program consisted of higher volumes and intensities in the first and third sessions of the week ( 3 sessions per week). Adjustments were made every three weeks, increasing volume and intensity on the first and third session and decreasing on the second session of the week, until nine weeks of training were completed. Despite significant improvements in dry-land strength (leg power and jumping ability), no significant improvements were found for flight distance when using any start technique. The authors suggested that improvements in jumping ability were not transferred to diving skills. Information on the periodization of different moments of this investigation was not provided. It would be of interest to know whether the teaching period of starts or the starts training were concurrent or not to the S\&C program. Nevertheless, the conclusions of this investigation must be analyzed with caution as the sample comprised non-swimmer subjects.

Summarizing the evidence of $\mathrm{S} \& \mathrm{C}$ training on starts and turns, we could conclude that plyometric training is the most 
effective in improving starts and turns. S\&C training ranging from six to eight weeks and with 2 sessions per week is suggested to improve performance. In each session subjects are allowed to perform between 1 to 6 sets and 1 to 10 repetitions, according to the chosen level of intensity. Rest between sets should vary from 60 to 90 seconds. This training program should be progressive, starting with low volumes and intensities to allow swimmers to adapt to plyometric training specificities. Moreover, exercises should be performed in as similar a way as possible to in-water movements. It would be of interest to analyze S\&C effects in older and more skilled swimmers. Transferability of S\&C gains to swimming performance remains controversial. Therefore, it would be of interest to compare plyometric training to other methods of S\&C training, and thus analyze the efficiency of different methods.

\section{Conclusion}

S\&C training in swimming is a common practice used by coaches and swimmers to enhance performance and to prevent injuries. Nevertheless, there is no consensus on the effectiveness of S\&C programs on swimming performance. Some limitations were found in the literature regarding this issue. Most investigations involved adolescent and adult swimmers, and S\&C programs with prepubescent swimmers are scarce and do not allow for valid conclusions. Younger swimmers should participate in S\&C programs, not only to enhance performance but also to build a solid foundation for preventing sports-related injuries. Moreover, few studies separated the samples and compared the gender effect, and those that did, gave no clear results. With regard to the type of S\&C training, maximal strength training is associated with swimming performance improvements in the oldest swimmers, particularly in relation to short distance ( 25 and $50 \mathrm{~m}$ ) races. However, it is questionable whether this is an adequate methodology to apply to younger swimmers and those at a lower competitive level. Weight lifting equipment is the most used training tool in research, nonetheless we must be aware that most swimming clubs may not have access to these resources. Additionally, young swimmers may not have the expertise and experience to use this equipment.

The influence of force exertion cannot be determined, as there were few investigations analyzing techniques other than freestyle. Likewise, the influence of S\&C in swimming distances above $200 \mathrm{~m}$ was not determined. Only one investigation assessed a middle distance $(400 \mathrm{~m})$ and presented positive results.

Based on this review, it is suggested low-volume S\&C training based on maximal strength, ranging from six to twelve weeks of 2 to 4 (approximately 24 sessions) sessions per week, for improving swimmers' performances. In each session, coaches should vary from 2 to 3 sets and with 3 to 5 repetitions, according to prescribed intensity. Rest intervals should vary between 2 to 5 minutes and the intensity should vary from 80 to $90 \%$ of $1 \mathrm{RM}$.

To improve starts and turns, a S\&C training ranging from six to eight weeks and with 2 sessions per week is suggested. In each session, swimmers should perform between 1 to 6 sets and 1 to 10 repetitions, according to the chosen level of intensity. Rest between sets should vary from 60 to 90 seconds. Volume should be progressive, such as raising intensity from low to high within the S\&C training program. It is recommended to start with low volumes and intensities to allow swimmers to adapt to plyometric training specificities. Only recommendations for adolescent swimmers were given, since no studies with older swimmers were included in this review.

Although there is a lack of coherent scientific evidence, S\&C training remains a commonly prescribed swimming practice. So, it seems fair to argue that further investigations should be carried out. To control for some gaps in protocols and to enable the generalizability of conclusions, randomized controlled trials (RCT) should be conducted. Future investigations should explore the following topics:

- differences in S\&C training effects induced by age and gender;

- effects of S\&C training in relation to different swimming techniques;

- effects of S\&C training in middle and long swimming distances;

- effects of S\&C training experimental periods over a season, to evaluate long-term effects;

- effects of a swimming adaption period after S\&C cessation to allow transferability of strength gains to swimming.

\section{Disclosure of interest}

The authors declare that they have no competing interest.

\section{Acknowledgements}

This project was supported by the National Funds through FCT - Portuguese Foundation for Science and Technology (UID/DTP/04045/2013) - and the European Fund for regional development (FEDER) allocated by European Union through the COMPETE 2020 Programme (POCl-01-0145FEDER-006969).

\section{References}

[1] Amaro NM, Marinho DA, Marques MC, Batalha NP, Morouço PG. Effects of dry-land strength and conditioning programs in age group swimmers. J Strength Cond Res 2017;31(9):2447-54.

[2] Girold S, Maurin D, Dugue B, Chatard JC, Millet G. Effects of dry-land vs. resisted- and assisted-sprint exercises on swimming sprint performances. J Strength Cond Res 2007;21:599-605.

[3] Haycraft J, Robertson S. The effects of concurrent aerobic training and maximal strength, power and swim-specific dryland training methods on swim performance: a review. J Aust Strength Cond 2015;23(2):91-9.

[4] Keskinen KL, Tilli LJ, Komi PV. Maximum velocity swimming: interrelationships of stroking characteristics, force production and anthropometric variables. Scand J Med Sci Sports 1989;11:87-92.

[5] Newton RU, Jones J, Kraemer WJ, Wardle H. Strength and power training of Australian olympic swimmers. Strength Cond J 2002;24(3):7-15.

[6] Morouço P, Keskinen KL, Vilas-Boas JP, Fernandes RJ. Relationship between tethered forces and the four swimming techniques performance. J Appl Biomech 2011;27:161-9. 
[7] Stager JM, Coyle MA. Energy Systems. In: Stager J, Tanner D, editors. Swimming - handbook of sports medicine and science. Massachusetts, United States of America: Blackwell Science; 2005. p. 1-19.

[8] Aspenes S, Kjendlie PL, Hoff J, Helgerud J. Combined strength and endurance training in competitive swimmers. J Sport Sci Med 2009;8:357-65.

[9] Bishop D, Smith R, Smith M, Rigby H. Effect of plyometric training on swimming block start performance in adolescents. J Strength Cond Res 2009;23(7):2137-43.

[10] Garrido N, Marinho DA, Reis VM, Van Den Tillaar R, Costa AM, Silva AJ, et al. Does combined dry land strength and aerobic training inhibit performance of young competitive swimmers? J Sport Sci Med 2010;9:300-10.

[11] Girold S, Jalab C, Bernard O, Carette P, Kemoun G, Dugué B. Dry-land strength training vs. electrical stimulation in sprint swimming performance. J Strength Cond Res 2012;26(2):497-505.

[12] Hong-Sun S, Dong-Ho P, Dong-Sik J. The effect of periodized strength training application on the Korea national team. Int J App Sports Sci 2009;21(2):122-45.

[13] Potdevin F, Alberty M, Chevutschi A, Pelayo P, Sidney M. Effects of a 6-week plyometric training program on performances in pubescent swimmers. J Strength Cond Res 2011;25(1):80-6.

[14] Strass D. Effects of maximal strength training on sprint performance of competitive swimmers. In: Ungerechts V, Wilke K, Reischle K, editors. Swimming Science. London, United Kingdom: Spon Press; 1988. p. 149-56.

[15] Weston M, Hibbs A, Thompson K, Spears I. Isolated core training improves sprint performance in national-level junior swimmers. Int J Sports Physiol Perform 2015;10(2):204-10.

[16] Sadowski J, Mastalerz A, Gromisz W, Niźnikowski T. Effectiveness of the power dry-land training programmes in youth swimmers. J Hum Kinet 2012;32:77-86.

[17] Morouço PG, Marinho DA, Amaro NM, et al. Effects of dry-land strength training on swimming performance: a brief review. J Hum Sport Exerc 2012;7(2):553-9.

[18] Tanaka H, Costill DL, Thomas R, Fink WJ, Widrick JJ. Dry-land resistance training for competitive swimming. Med Sci Sports Exerc 1993;25:952-9.

[19] Barbosa TM, Costa M, Marinho DA. Proposal of a deterministic model to explain swimming performance. Int J Swimming Kinetics 2013;2(1):1-54.

[20] Batalha N, Raimundo A, Tomas-Carus P, Paulo J, Simão R, Silva AJ. Does a land-based compensatory strength training program really influence the rotator-cuff balance of young competitive swimmers? Eur J Sport Sci 2015;15(8):764-72.

[21] Folland J, Williams A. The adaptations to strength training. Sports Med 2007;37(2):145-68.

[22] Leveritt M, Abernethy P, Barry BK, Logan PA. Concurrent strength and endurance training. A review. Sports Med 1999;28(6):413-27.

[23] Toussaint $H$, Truijens M. Power requirements for swimming a world-record $50 \mathrm{~m}$ front crawl. Int J Sports Physiol Perform 2006;1(1):61-4.

[24] Trappe S, Pearson DR. Effects of weight assisted dry-land strength training on swimming performance. J Strength Cond Res 1994;8:209-13.

[25] Breed R, Young W. The effect of a resistance-training programme on the grab, track and swing starts in swimming. J Sport Sci 2003;21(3):213-20.

[26] Cossor JM, Blanksby BA, Elliott BC. The influence of plyometric training on the freestyle tumble turn. J Sci Med Sport 1999;2(2):106-16.

[27] Manning J, Dooly-Manning C, Terrell D, Salas E. Effects of a power circuit weight training program on power production and performance. J Swim Res 1986;2(1):24-9.
[28] Barbosa TM, Costa M, Marinho DA, Coelho J, Moreira M, Silva AJ. Modeling the links between young swimmer's performance: energetic and biomechanical profiles. Pediatr Exerc Sci 2010;22:379-91.

[29] Morais JE, Silva AJ, Marinho DA, Marques MC, Batalha N, Barbosa TM. Modelling the relationship between biomechanics and performance of young sprinting swimmers. Eur J Sport Sci 2016;16(6):661-8.

[30] Kjendlie P, Stallman R, Stray-Gundersen J. Adults have lower stroke rate during submaximal front crawl swimming than children. Eur J App Physiol 2004;91(5-6):649-55.

[31] Tolfrey K. Responses to training. In: Armstrong N, editor. Paediatric exercise science: advances in sport and exercise science series. Edinburgh: Churchill Livingstone; 2007. p. 213-34.

[32] Faigenbaum AD, Kraemer WJ, Bimkie CJR, Jeffreys I, Micheli $\mathrm{LJ}$, Nitks $M$, et al. Youth resistance training: updated position statement paper from the national strength and conditioning association. J Strength Cond Res 2009;23(S5):60-79.

[33] Faigenbaum AD, Lloyd RS, MacDonald J, Myer GD. Citius, altius, fortius: beneficial effects of resistance training for young athletes. Br J Sports Med 2015;0:1-7.

[34] Engebretsen L, Steffen K, Bahr R, Broderick C, Dvorak J, Janarv $\mathrm{PM}$, et al. The International Olympic Committee consensus statement on age determination in high-level young athletes. Br J Sports Med 2010;44:476-84.

[35] Meylan C, Cronin J, Oliver J, Hopkins W, Contreras B. The effect of maturation on adaptations to strength training and detraining in 11-15-year-olds. Scand J Med Sci Sports 2014;24:156-64.

[36] Malina RM, Bouchard C, Bar-Or O. Growth, maturation and physical activity. 2nd ed. Champaign: Illinois: Human Kinetics; 2004.

[37] Seifert L, Barbosa TM, Kjendlie PL. Biophysical approach to swimming: gender effect. In: Davies SA, editor. Gender gap: causes, experiences and effects. New York, United States of America: Nova Science Publishers; 2010. p. 59-80.

[38] Barbosa TM, Bragada JA, Reis VM, Marinho DA, Carvalho C, Silva AJ. Energetics and biomechanics as determining factors of swimming performance: updating the state of the art. J Sci Med Sports 2010;13:262-9.

[39] Seifert L, Boulesteix L, Chollet D. Effect of gender on the adaptation of arm coordination in front crawl. Int J Sports Med 2004;25(03):217-23.

[40] Seifert L, Chollet D. Inter-limb coordination and constrains in swimming: a review. In: Beaulieu NP, editor. Physical activity and children: new research. New York, United States of America: Nova Science Publishers; 2008. p. 65-93.

[41] Bencke J, Damsgaard R, Saekmose A, Jorgensen P, Jorgensen $\mathrm{K}$, Klausen K. Anaerobic power and muscle strength characteristics of 11 years old elite and non-elite boys and girls from gymnastics, team handball, tennis and swimming. Scand J Med Sci Sport 2002;12:171-8.

[42] Bergeron MF, Mountjoy M, Armstrong N, Chia M, Côte J, Emery CA, et al. International Olympic Committee consensus statement on youth athletic development. Br J Sports Med 2015;49:843-51.

[43] Costill DL, King DS, Holdren A, Hargreaves M. Sprint speed vs. swimming power. Swim Tech 1983;20(1):20-2.

[44] Rohrs D, Mayhew J, Arabas C, Shelton M. The relationship between seven anaerobic tests and swim performance. J Swim Res 1990;6(4):15-9.

[45] Baker D, Wilson G, Carlyon B. Generality versus specificity: a comparison of dynamic and isometric measures of strength and speed-strength. Eur J Appl Physiol 1994;68(4):350-5.

[46] Barbosa TM, Morais JE, Marques MC, Costa MJ, Marinho DA. The power output and sprinting performance of young swimmers. J Strength Cond Res 2015;29(2):440-50. 
[47] Toussaint HM. Strength power and technique of swimming performance: science meets practice. In: Leopold W, editor. Schwimmen Lernen und Optimieren. Beucha, Deutschland: Schwimmtrainer-Vereinigung V; 2007. p. 43-54.

[48] González-Badillo JJ, Sánchez-Medina L. Movement velocity as a measure of loading intensity in resistance training. Int J Sports Med 2010;31(5):347-52.

[49] Häkkinen K, Pakarinen A, Kyrolainen H, Cheng S, Kim DH, Komi PV. Neuromuscular adaptations and serum hormones in females during prolonged power training. Int J Sports Med 1990;11:91-8.

[50] Wilmore J, Costill D. Physiological adaptations to physical training. In: Dubuque IA, Brown WMC, editors. Training for sport and activity, Chapter 11. 1988. p. 113-212.

[51] Bosquet L, Berryman N, Dupuy O, Mekary S, Arvisais D, Bherer $L$, et al. Effect of training cessation on muscular performance: a meta-analysis. Scand J Med Sci Sports 2013;23:140-9.
[52] Willardson JM. Core stability training: applications to sports conditioning programmes. J Strength Cond Res 2007;21(3):979-85.

[53] Linnamo V, Häkkinen K, Komi P. Neuromuscular fatigue and recovery in maximal compared to explosive strength loading. Eur J Appl Physiol 1997;77:176-81.

[54] Arellano R, Sanchez-Molina J, Navarro F, De Aymerich J. Analysis of $100 \mathrm{~m}$ backstroke, breaststroke, butterfly, freestyle swimmers at the 2001 European Youth Olympic days. In: IX Biomechanics and Medicine in Swimming. University of Saint Etienne; 2003. p. 255-60.

[55] Thayer AL, Hay JG. Motivating start and tum improvement. Swim Tech 1984;20(4):17-20. 